\title{
Targeted agents for advanced thyroid cancer: "knowledge is power" - the role of multikinase inhibitors
}

\author{
"...the understanding of the molecular pathogenesis of thyroid cancers \\ paved the way for the development of novel cutting-edge therapeutic \\ agents; for this reason, a more tailored disease management will be \\ possible."
}

\begin{abstract}
Alberto Mangano ${ }^{* 1,2}$, Hoon Yub Kim ${ }^{3}$, Georgios D Lianos ${ }^{4,5}$, Dimitrios H Roukos $^{4,5}$ \& Gianlorenzo Dionigi ${ }^{1,2}$
\end{abstract}

Thyroid cancer is the most prevalent neoplasm among endocrine tumors, presenting an increasing incidence trend over the last few years $[1,2]$. The highest incidence is among female patients [1]. Thyroid cancers can be histopathologically classified into: anaplastic thyroid carcinoma (2\% of all thyroid cancers), medullary thyroid carcinoma (MTC; 4\%) and differentiated thyroid carcinoma (DTC; 94\%), which represents he vast majority of thyroid cancers and presents three subtypes: Hurthle cell carcinoma (3\%), follicular thyroid carcinoma $(11 \%)$ and papillary thyroid carcinoma (PTC; 80\%) [3]. Notably, even if the anaplastic thyroid carcinoma represents just a small minority of all thyroid cancers, it is responsible for up to $50 \%$ of deaths [4].

De facto, early-stage thyroid cancers (i.e., the vast majority) have an excellent outcome and surgery often represents the gold-standard therapy. In particular, total thyroidectomy is mostly indicated in these cases [5]. The surgical technique should be standardized and, of note, a standardized intraoperative neuromonitoring of the recurrent laryngeal nerve is a relevant technological adjunct [6,7]. In fact, after therapy in cases of DTC the outcome is excellent, with a 5-year overall survival rate of nearly $98 \%$. Not withstanding these positive results, 10 years after treatment distant metastases can unfortunately develop in one patient out of ten and there is a relevant local recurrence rate (up to two patients out of 20) [8].

On the contrary, relapsed thyroid cancers often evolve towards metastatic forms and a related loss of iodine avidity, with a poor response rate to the standard therapies and an unfavorable outcome [4]. Doxorubicin is currently the only systemic cytotoxic agent approved for DTC, but unfortunately it has relevant adverse events and a poor response rate [4].

Since traditional cytotoxic drugs show poor results, new treatment options are needed. The in-depth understanding of

\section{KEYWORDS}

- advanced thyroid cancer

- intertumor heterogeneity

- intratumor heterogeneity

- multikinase inhibitor • targeted agent

"Thyroid cancer is the most prevalent neoplasm among endocrine tumors, presenting an increasing incidence trend over the last few years." 
"Multikinase inhibitors have shown improved clinical outcome and implemented tumor response in advanced thyroid cancer.” the molecular basis of thyroid cancer tumorigenesis is mandatory for the development and implementation of novel, targeted and effective agents. Hence, over the last few years these molecular processes have been extensively studied and better clarified. For this reason, the development of new targeted molecules for the advanced and refractory disease therapy has been possible. In fact, two targeted treatments have been approved so far: cabozantinib [9] and vandetanib [10].

Many novel agents are being evaluated for advanced thyroid cancer [3]. This editorial specifically focuses on multikinase inhibitors, agents that can block pathways essential for cancer survival and spread. Our aim is to briefly review the latest therapy advancements, providing our own additional remarks on this topic.

\section{Thyroid carcinoma: molecular pathways}

Tyrosine kinases (TKs) are phosphorylative enzymes involved in cellular signal transduction pathways important in oncological contexts [11]. Tumoral constitutive activation of oncogenic signaling can be related to some genetic alterations that lead to mutation/overexpression of several intracellular signals (including TKs) $[12,13]$. Thyroid cancers are often characterized by genetic alterations activating the PI3K-AKT-mTOR pathway and the RET/ PTC-RAS-RAF-MAPK axis. Notably, RET can activate a wide variety of signaling cascades (including PI3K-AKT and MAPK) [12,13]. The more aggressive types of thyroid cancer often present alterations in PI3K-regulated intracellular signals [12,13]. RAS mutations and PAX8/ PPAR- $\gamma$ rearrangements are the most frequent alterations in follicular thyroid carcinoma [12]. However, most PTCs present mutations of $R A S$ and $B R A F$ genes and RET/PTC rearrangement $[12,13]$. What is more, sporadic MTC often shows a cross-activation of the PI3K and MAPK pathways related to amplification of RAS-RAF signaling $[12,13]$. Moreover, other tyrosine kinase receptors activating the same pathways have been shown: the HGF receptor encoded by the c-Met (MET) proto-oncogene EGF receptor, the VEGF receptor family and the FGF receptor family $[12,13]$.

\section{TKs \& resistance}

Tumors can rapidly adapt and drug resistance may develop; thus, the benefits of tyrosine kinase receptor inhibitors can be transient/absent [14].
Two resistance mechanisms have been shown for the VEGF pathway inhibitors: evasive, linked to the ability of the cancer to avoid angiogenetic blockage via several mechanisms and intrinsic, due to pre-existing cancer microenvironment conferring indifference to the inhibitor [14]. Since the majority of solid tumors present deregulation of multiple pathways, current drug development tends toward development of a multikinase inhibitor, such as a single agent endowed with the potential of multiple targeting [12].

\section{Thyroid carcinoma \& multikinase inhibitors}

Multikinase inhibitors have shown improved clinical outcome and implemented tumor response in advanced thyroid cancer [4]. The effectiveness of these drugs may be linked to their capability to simultaneously inhibit multiple pathways and vascular sprouting [12]. Some of these drugs are now under clinical investigation in patients with MTC and DTC, whereas some others have already been approved for advanced MTC.

Cabozantinib inhibits RET, VEGFR-2 and the HGF receptor. Its clinical role for advanced MTC was observed in a Phase I study [15] and subsequently in a Phase III study in metastatic MTC [9], where the objective response rate reported was $0 \%$ for placebo and $28 \%$ for cabozantinib. Median progression-free survival was 4 months for placebo and 11.2 months for cabozantinib. What is more, progression-free survival at 1 year was reported to be $7.2 \%$ for placebo and $47.3 \%$ for cabozantinib. Adverse events were manageable and non-life-threatenening [9]. The results of the Phase III trial led to US FDA approval of a multikinase inhibitor for patients with progressive metastatic MTC.

Vandetanib blocks VEGFR-1, VEGFR-2, the EGF receptor and RET-TK. Two Phase II trials $[16,17]$ investigated vandetanib in hereditary MTC, with promising results that led to a Phase III trial [10] in advanced hereditary or sporadic MTC using vandetanib versus placebo. This study demonstrated a significantly prolonged progression-free survival on vandetanib and a significantly major objective response rate of 45 and $1 \%$, respectively. The most frequent adverse events were non-life-threatenening and manageable. This Phase III study led to the FDA and the EMA approval of vandetanib for progressive or metastatic MTC in adults.

Lenvatinib (E7080) is a multiple tyrosine kinase receptor inhibitor targeting FGFR-1-4, 
PDGFR $\beta 56$, VEGFR-1-3, RET and c-Kit. The role of this promising agent has been recently reviewed by Stjepanovic and Capdevilla [12] The Phase III SELECT trial is a multicenter, randomized, double-blind, placebo-controlled study of lenvatinib in radioiodine-refractory DTC patients. The primary end points are overall survival, progression-free survival and secondary objective response rate. Recently it has been shown that the SELECT trial has fulfilled the primary end point in terms of progression-free survival benefit in the therapy of radioiodine-refractory DTC. Consequently, lenvatinib has been submitted for market authorization [12].

Sorafenib is an inhibitor of VEGFR-1, VEGFR-2, VEGFR-3, PDGFR, RET, RAF and c-Kit. After showing promising results, this agent has been studied in a double-blind Phase III study [18] comparing placebo versus radioiodinerefractory DTC. There was a notable improvement in median progression-free survival, stable disease, objective response and disease control rate for sorafenib versus placebo. What is more, $73 \%$ of patients showed some degree of reduction in target tumor lesions. The most frequent adverse events were non-life-threatenening and manageble [18].

Finally, some other interesting agents are currently under investigation in Phase II trials: axitinib; sunitinib; motesanib diphosphate; and pazopanib. However, for these last aforementioned molecules more data, from Phase III trials, are needed.

\section{Discussion}

Early-stage thyroid cancers have an excellent outcome and total thyroidectomy often represents the gold-standard therapy [5,19]. Unfortunately, just a few effective therapeutic tools have been available for advanced stages so far. However, the understanding of the molecular pathogenesis of thyroid cancers paved the way for the development of novel cutting-edge therapeutic agents; for this reason, a more tailored disease management will be possible. Not withstanding the aforementioned promising tools (in particular multikinase inhibitors), there are still some relevant challenges to be faced.

De facto, cancer is still an enigmatic, complex, heterogeneous, rapidly evolving, rapidly shifting, fast adapting and unfortunately not completely understood enemy. In particular, over the last few years, technological improvements have allowed an analysis of individual cancer genomes at single nucleotide level [20]. Moreover, beside the well known intertumor heterogeneity between cancers of the same histopathological type, there has recently been much more new evidence for intratumor heterogeneity (ITH) in terms of differences in spatially separated areas of the same tumor or in its metastatic sites and, of note, within individual cancer biopsies (i.e., a single biopsy may underevaluate the cancer mutational landscape) [20]. Notably, beside a spatial evolution, there is a temporal evolution of ITH [20]. In this way, another element to be clarified in the future is if the theoretical Darwinian selective pressure of cytotoxic therapy could have a role in enhancing ITH; its potential clinical consequences should be better clarified [20]. Moreover, another element to be investigated in the future is the relationship between ITH and drug resistance development. In this way, the advent of new technology to continuously monitor the cancer subclonal dynamics could be useful [20].

At the same time, beside the necessary indepth knowledge of cancer dynamics and the development of more accurate tools for ITH monitoring (to understand which molecule is the most effective in that precise moment for that specific cancer), it is also mandatory to create new therapeutic tools in order to limit such a Darwinian adaptive nature of cancer. In this way it will become more easy to understand which drug should be used first and how to overcome a possible resistance to therapy.

In conclusion, "knowledge is power" (Sir Francis Bacon, Meditationaes Sacrae, 1597) and if we will better clarify those mechanisms, we should be able to precisely select (using standardized parameters) which patients will benefit the most (in that particular moment and in a personalized way) from the targeted agent specifically developed.

\section{Financial \& competing interests disclosure}

The authors have no relevant affiliations or financial involvement with any organization or entity with a financial interest in or financial conflict with the subject matter or materials discussed in the manuscript. This includes employment, consultancies, honoraria, stock ownership or options, expert testimony, grants or patents received or pending, or royalties.

No writing assistance was utilized in the production of this manuscript. “...cancer is still an enigmatic, complex, heterogeneous, rapidly evolving, rapidly shifting,

fast adapting and

unfortunately not completely understood enemy." 


\section{References}

1 Divies L, Welch HG. Current thyroid cancer trends in the United States. JAMA

Otolaryngol. 140(4), 317-322 (2014).

2 Thyroid cancer incidence statistics. www.cancerresearchuk.org

3 National Comprehensive Cancer Network. Guidelines for thyroid carcinoma. www.nccn.org

4 Salama JK, Golden DW, Yom SS et al. Appropriateness criteria thyroid carcinoma. Oral Oncol. 50, 577-586 (2014).

5 Billimoria KY, Bentrem DJ, Ko CY et al. Extent of surgery affects survival for papillary thyroid cancers. Ann. Surg. 246(3), 375-381 (2007).

6 Dionigi G, Van Slycke S, Boni L et al. Limits of neuromonitoring in thyroid surgery. Ann. Surg. 258(1), e1-e2 (2013).

7 Frattini F, Mangano A, Boni L, Rausei S, Biondi A, Dionigi G. Intraoperative neuromonitoring for thyroid malignancy surgery: technical notes and results from a retrospective series. Updates Surg. 62, 183-187 (2010).

8 Yang L, Shen W, Sakamoto N. Population based study evaluating and predicting the probability of death resulting from thyroid cancer and other causes among patients with thyroid cancer. J. Clin. Oncol. 31(4), 68-74 (2013).

9 Elisei R, Schlumberger MJ, Müller SP et al. Cabozantinib in progressive medullary thyroid cancer. J. Clin. Oncol. 31(29), 3639-3646 (2013).

10 WellsSAJr, Robinson BG, Gagel RF et al. Vandetanib in patients with locally advanced or metastatic medullary thyroid cancer: a randomized, double-blind Phase III trial. J. Clin. Oncol. 30(2), 134-141 (2012).

11 Krause DS, Van Etten RA. Tyrosine kinases as targets for cancer therapy. N. Engl. J. Med. 353(2), 172-187 (2005).

12 Stjepanovic N, Capdevila J. Multikinase inhibitors in the treatment of thyroid cancer: specific role of lenvatinib. Biologics 8 , 129-139 (2014).

13 Omur O, Barn Y. An update on molecular biology of thyroid cancers. Crit. Rev. Oncol. Hematol. 90 (3), 233-252 (2014).

14 Bergers G, Hanahan D. Modes of resistance to anti-angiogenic therapy. Nat. Rev. Cancer 8(8), 592-603 (2008).

15 Kurzrock R, Sherman SI, Ball DW et al. Activity of XL184 (cabozantinib), an oral tyrosine kinase inhibitor, in patients with medullary thyroid cancer. J. Clin. Oncol. 29(19), 2660-2666 (2011).

16 Wells SA Jr, Gosnell JE, Gagel RF et al. Vandetanib for the treatment of patients with locally advanced or metastatic hereditary medullary thyroid cancer. J. Clin. Oncol. 28(5), 767-772 (2010).

17 Robinson BG, Paz-Ares L, Krebs A, Vasselli J, Haddad R. Vandetanib $(100 \mathrm{mg})$ in patients with locally advanced or metastatic hereditary medullary thyroid cancer. J. Clin. Endocrinol. Metab. 95(6), 2664-2671 (2010).

18 Brose MS, Nutting C, Jarzab B et al. Sorafenib in locally advanced or metastatic patients with radioactive iodine-refractory differentiated thyroid cancer: the Phase III DECISION trial. J. Clin. Oncol. 31(Suppl.), Abstract 04 (2013).

19 Dionigi G, Dionigi R, Bartalena L, Boni L, Rovera F, Villa F. Surgery of lymph nodes in papillary thyroid cancer. Expert Rev. Anticancer Ther. 6(9), 1217-1229 (2006).

20 Swanton C. Intratumour heterogeneity: evolution through space and time. Cancer Res. 72(19), 4875-4882 (2012). 